\title{
On time
}

\section{INTRODUCTION}

Time, its use and its boundaries, pervades and underpins almost all encounters in primary care. Patients, GPs and therapists all ask for more - for longer consultations or for more sessions. In this article we shall try to examine what this wished-for quality represents and what it might symbolise.

\section{PATIENTS WANT MORE TIME}

Health service surveys are notorious for providing favourable results; suggesting that patients tend to view the NHS positively overall. This may arise from a reluctance to antagonise the care-giver or from a level of expectation fixed by past experience - we learn to expect a certain level of care and accept it. Although such mindsets might mitigate attitudes toward the health services in general, patients make fewer allowances when judging their interaction with individual doctors:

'While patients might acknowledge that physicians do not have a lot of time at their disposal, they expect their own individual cases to receive the attention they deserve. Such common remarks as "He doesn't listen to me", "She didn't take time to examine me", "How can he know what l've got?" and "She's always busy," indicate dissatisfaction with quality of time spent with physicians'. ${ }^{1}$

Notably, the authors refer to 'dissatisfaction with quality of time', rather than length of time. The distinction is important. Patients seem to desire quality time, but quality is difficult to define and hard to commodify. Quantity of time, however, is far more easily traded and may be marketed as a proxy for quality.

\section{PATIENTS WILL PAY FOR TIME}

One illustration of the time-for-money medical economy is seen in the effect of different healthcare systems on consultation length. In a survey of six European countries Deveugele et $a^{2}$ found that in four countries the mean GP consultation length was 10 minutes or less.

In Switzerland and Belgium, however, the average length of consultation was found to be 15 minutes. The authors note:

\begin{abstract}
'Moreover, GPs in Belgium and Switzerland are paid mostly by direct payment from the patient at the end of the consultation. This creates a relation between the doctor and the patient that is based on "value for money" or, ultimately, "time for money" for patient and doctor'. ${ }^{2}$
\end{abstract}

This willingness to pay would seem to support the contention that it is more time by itself that patients want. Correspondingly, this is what doctors feel they need to give in a competitive free market for primary care: time for money. However, we must remain mindful of our earlier acknowledgement that quantity of time - more readily conceptualised and commodified - may simply be a surrogate for the more prized, but less tangible quality.

\section{PATIENTS USE QUANTITY OF TIME AS A SURROGATE MEASURE OF QUALITY (EMOTIONAL FACTORS AND FAVOURABLE EXPERIENCE)}

The proposition that quantity serves as the patients' proxy marker of quality is evidenced by the fact that patients' retrospective perceptions of consultation duration correlate with satisfaction.

Emotional factors and a favourable experience of a visit can influence the evaluation of its duration. In one study the most satisfied patients tended to overestimate the length of the consultation, while those who were less satisfied underestimated it. ${ }^{1}$ This finding has been replicated $^{3}$ and it has been found that underestimation was associated with the emotional component of the consultation and unrelated to other aspects such as information giving or examination. It was also found that lower levels of satisfaction were correlated with a lower intention to comply with the doctors' recommendations. The authors conclude:
'Patients' dissatisfaction with consultation length could be changed by making consultations longer. Alternatively, it could also be managed by changing how a given time is spent. In particular, a doctor who listens and tries to understand their patient may make the patient feel more satisfied with consultation length and subsequently more motivated to follow any recommendations for change. ${ }^{3}$

It seems clear that a favourable experience, and one that is likely to be more effective through greater adherence to treatment or advice, does not depend on duration of the consultation so much as the content of it. More evidence of patients using their perception of the time spent with a doctor as a measure of the doctor's receptiveness comes from a recent study of inpatients. Greater understanding of their condition and greater satisfaction with their care, as well as a perception that the doctor spent $40 \%$ more time with them, was found when the doctor sat rather than stood during the consultation. ${ }^{4}$

\section{HOW THEN HAS THIS COME TO PASS? THAT OUR PATIENTS CAN BE SOLD QUANTITY WHEN THEY WANT QUALITY? AND THAT THEY PERCEIVE 'MORE' WHEN WE GIVE THEM 'BETTER'?}

Time is the doctor's most visible - and visibly lacking - resource. Lussier and Richard ${ }^{1}$ point out some of the cues that tell patients about the limited time available for them; 'packed waiting rooms, busy physicians and staff, and the frenzied pace of office functioning'. That doctors broadcast these signals of being unable to give time as freely as patients may want may not be entirely accidental and may have a significance not previously acknowledged. To be busy is to be important; being important and being needed increases the power of the physician over their supplicants in the waiting room. With time as the essence of this power differential, it may be no 
coincidence that patients demand our time to redress the balance. And since time seems our most precious resource, little wonder that this is how our worth is measured.

Also, to at least some extent, the perceived associations of quality and quantity are real. Shorter consultations are associated with higher rates of antibiotic prescribing. ${ }^{5} \quad$ Conceivably, swifter consultations are less patient-centred, more biomedical and hence involve more prescriptions for physical symptoms. By contrast, a consultation in which psychosocial issues are addressed is likely to last longer. Patients seem to be expressing a wish to buy out of the former and into the latter consultation. Time is simply the currency, the unit in which that favourable perception, emotional content and intangible quality of a (patient-centred) consultation is measured.

The tangible health benefits of the intangible quality consultation are clear: not only are listened-to patients more motivated to follow any recommendations for change ${ }^{3}$ but, also, a recent study ${ }^{6}$ has demonstrated a connection between the quality of a consultation, measured by its degree of empathy, and the duration of symptoms using the common cold as a model. Those patients that recorded perfect empathy scores for their consultations had significantly shorter illnesses both measured by symptom scores and, highly relevantly and importantly, by a measure of their immune response (the level of the immune cytokine interlukin-8 recovered from nasal washes). The degree of connectedness perceived by patients in the consultation was directly linked to their immune responses. Michael Balint was ahead of his time when he coined the expression 'the drug doctor':? this study illustrates that 'doctor', when correctly administered, has antiviral properties and seems to be equipotent with Tamiflu ${ }^{\circledR}$. Although consultation length is not reported, what the patients perceived as happening within the consultation in terms of empathy was the significant factor in this study.

\section{TIME NOT SO EASILY OBTAINED}

Speed and a biomedical approach are more accurately understood as features of a doctor than of a consultation of certain duration. If they had longer consultations these doctors might still be the type of practitioners who believe in pharmacology over exploration. One can speculate that this is defence and by keeping a very medical agenda they avoid some of the difficult, possibly uncomfortable, emotional and often insoluble, content that patients sometimes bring to consultations.

The lengthy consultation too is largely doctor-determined. In the study of primary care consultations in six European countries it was found that:

'The presence of psychosocial problems in the consultation was an important factor influencing the length of the consultation, and doctors' and patients' perceptions about psychosocial problems affected the length of the consultation. The difference between the effects of the doctors' and the patients' perceptions was remarkable. When doctors perceived a psychosocial problem, the duration of the consultation increased; this was as true when the doctor thought the psychosocial element was important, even if it was not mentioned [in] during the consultation, as when he or she made a psychosocial diagnosis. From the patient's perspective, the consultation time was longer when the patient expected some help on psychosocial aspects from the doctor than when they did not. The length of consultation was not significantly longer for consultations in which psychosocial aspects were given as a reason for the encounter by the patient. ${ }^{2}$

This clearly illustrates that it is the doctor and his or her perception of the problem being presented that controls the length of time given to it.

It is a methodological weakness of all the studies that doctors, and to a lesser extent patients, cannot be randomised in a trial to provide the answer to the questions about whether longer consultations lead to better outcomes and greater satisfaction. The way a doctor consults may be a fixed marker for certain attributes that may not be amenable to change by extending consultation length. Notwithstanding this limitation a number of observational studies have been reported. In a systematic review it was found that while there was an increase in recording blood pressure and smoking status, there was no evidence that changing to longer appointments led to greater patient satisfaction. $^{8}$

This review also looked at doctor stress and found some evidence of reduced stress with longer consultations. The finding that it is the doctor who might benefit from longer consultations is echoed in a report of a working party of the RCGP who found that longer consultations led to greater professional satisfaction and, if these longer consultations were combined with more realistic scheduling, to reduced stress levels. ${ }^{9}$ It seems, therefore, that it may be in the doctor's and not the patient's direct interest to have longer appointments, but indirectly a more satisfied and less stressed doctor has some advantages.

When considering the barriers preventing doctors from consulting in a manner that gives better outcomes in both compliance and satisfaction, and is therefore likely to give more satisfaction to the doctor, we cannot disregard external factors. Doctors have to balance competing and ever-increasing demands, and they may be 'activity trapped' and unable to make the initial investment in changing consultation style. They have to hear stories of loss and even death, and contain patients' anxiety, on a daily basis.

The nature of the role of GPs makes it unsurprising that defences play a part too. Menzies Lyth's classic study of nurses and their anxiety ${ }^{10}$ has some resonances with GPs. Although the tasks and contact may be different there are parallels and the 
following passage only needs a little adaption to be equally true for GPs:

'Nursing patients who have incurable diseases is one of the nurse's most distressing tasks. Nurses are confronted with the threat and the reality of suffering death as few lay people are. Their work involves carrying out tasks which, by ordinary standards, are distasteful, disgusting and frightening. Intimate physical contact with patients arouses strong libidinal and erotic wishes and impulses that may be difficult to control. The work situation arouses very strong and mixed feelings in the nurse: pity, compassion and love; guilt and anxiety; hatred and resentment of the patients who arouse these strong feelings; envy of the care given to the patient. ${ }^{10}$

In medicine there is no culture of supervision, and self-reliance, including emotional self-reliance, is prized. These facts make an examination of the psychic barriers to change important. Given the expectation that they should care without being cared for, resentment and envy can arise. And one of the (unrecognised) envies that GPs may experience is the time and care they perceive patients receiving from therapists. Dealing with this and other difficult emotions may lead to detachment and denial of feelings.

Counsellors who work in primary care operate a very different system of time allocation. Their appointments are (by comparison) long, but constant and of a set duration. Access is by referral and additional appointments between scheduled sessions cannot usually be made. Under the NHS a fixed number of sessions is set at the outset. There is usually no ongoing contact after the end of the agreed sessions. These are all differences in the therapeutic frame but perhaps the greatest difference is in the content of sessions. While psychosocial and emotional factors are important in GP consultations they can sometimes be seen as peripheral to the medical content. In counselling sessions they are the main content. Asking for more time, either within sessions or for more sessions, will be a familiar scenario. Time and attention are again linked. There is scope for considerable envy of counsellors by doctors and this could be a factor in relationships. The long, measured appointments of a self-selected group of referred patients contrasts with the world of most GPs with open, but time-limited access in which all comers must be seen. Resorting to being busy, and therefore in demand and important, may be a response. For the patient there may be echoes of archetypal family roles: the busy, unengaged dad who 'fixes' things and the more present mother who takes care.

In summary it seems time is the currency in which we trade. We can deduce from the evidence that patients overestimate the length of consultation when there seems to be a connection, that is to say they give it more currency, that time is a symbol representing some other wished-for commodity. Connecting will largely be an unconscious act and it is perhaps not surprising that it seems more valuable - to have greater time value, to be longer - in the conscious, concrete world. But time is finite and the trade off between time and connection cannot ever be perfect. Like any other precious currency, variations and distortions in supply, quality and demand can lead to frictions. In the case of primary care, the differing perceptions of doctors, therapists and patients can contribute to these distortions but ultimately time seems to be a cipher for connectiveness.

\section{Richard Stevens and Ann Mountford}

\section{Acknowledgements}

We are grateful to Andrew Elder, Maria Ward and especially to Andrew Moscrop for the comments and suggestions they made to this manuscript. This paper was presented at a seminar of the Primary Care Section of the association for Psychoanalytic Psychotherapy in November 2009.

\section{REFERENCES}

1. Lussier M-T, Richard C. Time flies; patients' perceptions of consultation length and actual duration. Can Fam Physician 2007; 53(1): 46-47.

2. Deveugele M, Derese A, van den Brink-Muinen A, et al. Consultation length in general practice: cross sectional study in six European countries. BMJ 2002; 325: 472.

3. Ogden J, Bavalia K, Bull M, et al. 'I want more time with my doctor': a qualitative study of time and the consultation. Fam Pract 2004; 21(5): 479-483.

4. The University of Kansas Hospital. Sitting down on the job: new data finds that patients are happier when doctors sit down, even if they don't stay as long. http://www.kumed.com/default.aspx?id=5350 (accessed 14 May 2010).

5. Wang K, Seed P, Schofield P, et al. Which practices are high antibiotic prescribers? Br J Gen Pract 2009; 59: 724-727.

6. Rakel D, Hoeft T, Barrett B, et al. Practitioner empathy and the common cold. Fam Med 2009; 41(7): 494-501.

7. Balint M. The doctor, his patient and the illness. London: Pittman, 1957.

8. Wilson A, Childs S. The effect of interventions to alter the consultation length of family physicians: a systematic review. Br J Gen Pract 2006: 56(532): 876-882.

9. Freeman G, Horder J, Howie J, et al. Evolving genera practice consultation in Britain: issues of length and context. BMJ 2002; 324: 880-882.

10. Menzies Lyth, I. The functions of social systems as a defence against anxiety: a report on a study of the nursing service of a general hospital. Human Relations 1959; 13: 95-121.

DOI: 10.3399/bjgp10X509720 